\title{
Minimalism in Cryptography: The Even-Mansour Scheme Revisited
}

\author{
Orr Dunkelman ${ }^{1,2}$, Nathan Keller ${ }^{2,3}$, and Adi Shamir ${ }^{2}$ \\ 1 Computer Science Department \\ University of Haifa \\ Haifa 31905, Israel \\ orrd@cs.haifa.ac.il \\ 2 Faculty of Mathematics and Computer Science \\ Weizmann Institute of Science \\ P.O. Box 26, Rehovot 76100, Israel \\ \{nathan.keller, adi.shamir\}@weizmann.ac.il \\ 3 Department of Mathematics \\ Bar-Ilan University \\ Ramat Gan 52900, Israel
}

\begin{abstract}
In this paper we consider the following fundamental problem: What is the simplest possible construction of a block cipher which is provably secure in some formal sense? This problem motivated Even and Mansour to develop their scheme in 1991, but its exact security remained open for more than 20 years in the sense that the lower bound proof considered known plaintexts, whereas the best published attack (which was based on differential cryptanalysis) required chosen plaintexts. In this paper we solve this open problem by describing the new Slidex attack which matches the $T=\Omega\left(2^{n} / D\right)$ lower bound on the time $T$ for any number of known plaintexts $D$. Once we obtain this tight bound, we can show that the original two-key Even-Mansour scheme is not minimal in the sense that it can be simplified into a single key scheme with half as many key bits which provides exactly the same security, and which can be argued to be the simplest conceivable provably secure block cipher. We then show that there can be no comparable lower bound on the memory requirements of such attacks, by developing a new memoryless attack which can be applied with the same time complexity but only in the special case of $D=2^{n / 2}$. In the last part of the paper we analyze the security of several other variants of the Even-Mansour scheme, showing that some of them provide the same level of security while in others the lower bound proof fails for very delicate reasons.
\end{abstract}

Keywords: Even-Mansour block cipher, whitening keys, minimalism, provable security, tight security bounds, slide attacks, slidex attack.

\section{Introduction}

A major theme in cryptographic research over the last thirty years was the analysis of minimal constructions. For example, many papers were published on 
the minimal cryptographic assumptions which are necessary and sufficient in order to construct various types of secure primitives. Other examples analyzed the smallest number of rounds required to make Feistel structures with truly random functions secure, the smallest possible size of shares in various types of secret sharing schemes, and the simplest way to transform one primitive into another by using an appropriate mode of operation. Since the vague notion of conceptual simplicity only partially orders all the possible schemes, in many cases we have to consider minimal schemes (which are local minima that become insecure when we eliminate any one of their elements) rather than minimum schemes (which are global minima among all the possible constructions).

In the case of stream ciphers, one can argue that the simplest possible secure scheme is the one-time pad, since any encryption algorithm requires a secret key, and XORing is the simplest conceivable way to mix it with the plaintext bits. The question we address in this paper is its dual: What is the simplest possible construction of a block cipher which has a formal proof of security?

This problem was first addressed by Even and Mansour 89] in 1991. They were motivated by the DESX construction proposed by Ron Rivest in 1984 [15], in which he proposed to protect DES against exhaustive search attacks by XORing two independent prewhitening and postwhitening keys to the plaintext and ciphertext (respectively). The resultant scheme increased the key size from 56 to 184 bits without changing the definition of DES and with almost no additional complexity. The Even-Mansour scheme used such whitening keys but eliminated the keyed block cipher in the middle, replacing it with a fixed random permutation that everyone can share. The resultant scheme is extremely simple: To encrypt a plaintext, XOR it with one key, apply to it a publicly known permutation, and XOR the result with a second key.

To argue that the Even-Mansour scheme is minimal, its designers noted in [9] that eliminating either one of the two XORed keys makes it easy to invert the known effect of the permutation on the plaintext or ciphertext, and thus to recover the other key from a single known plaintext/ciphertext pair. Eliminating the permutation is also disastrous, since it makes the scheme completely linear. In fact, the two-key EM block cipher is not minimal in the sense that it can be further simplified into a single-key variant with half as many key bits which has exactly the same security.

To compare various variants of the Even-Mansour scheme, we need tight bounds on the exact level of security they provide. Unfortunately, all the bounds published so far are not tight in the sense that the lower bound allows known message attacks whereas the best known upper bounds require either chosen plaintexts or an extremely large number of known plaintexts.

One of the main tools used in previous attacks was the slide attack 3. Originally, slide attacks were developed in order to break iterated cryptosystems with an arbitrarily large number of rounds by exploiting their self similarity under small shifts. The attack searched the given data for a slid pair of encryptions which have identical values along their common part (see Section 3.2 for formal definitions). For each candidate pair, the attack uses the two known plaintexts 
and two known ciphertexts to analyze the two short non-common parts in order to verify the assumption that the two encryptions are indeed a slid pair, and if so to derive some key material. A different variant of this attack, called slide with a twist [4, tries to find a slid pair consisting of one encryption and one decryption, which have identical values along their common parts (i.e., the attack considers both shifts and reversals of the encryption rounds). In both cases, the existence of slid pairs is a random event which is expected to have a sharp threshold: Regardless of whether we use known or chosen messages, we do not expect to find any slid pairs if we are given fewer than $2^{n / 2}$ encryptions where $n$ is the size of the internal state 1 Consequently, we cannot apply the regular or twisted slide attack unless we are given a sufficiently large number of encryptions, even if we are willing to trade off the lower amount of data with higher time and space complexities.

In this paper we propose the slidex attack, which is a new extended version of the slide attack that can efficiently use any amount of given data, even when it is well below the $2^{n / 2}$ threshold for the existence of slid pairs. Its main novelty is that we no longer require equality between the values along the common part, but only the existence of some known relationship between these values. By using this new attack, we can finally close the gap between the upper and lower bounds on the security of the Even-Mansour scheme.

To demonstrate the usefulness and versatility of the new slidex attack, we apply it to several additional schemes which are unrelated to Even-Mansour. In particular, we show how to break 20 rounds of GOST using $2^{33}$ known plaintexts in $2^{77}$ time. In the extended version of this paper [7] we show several additional attacks, such as how to use the complementation property of DES in order to attack it with a slide attack even when it is surrounded by Vaudenay's decorrelation modules.

The paper is organized as follows. In Section[2 we introduce the Even-Mansour scheme, describe its formal proof of security, and survey all the previously published attacks on the scheme. In Section 3 we describe the known types of slide attacks, and explain why they cannot efficiently exploit a small number of known plaintexts. We then introduce our new Slidex attack, and use it to develop a new upper bound for the security of the Even-Mansour scheme which matches the proven lower bound for any number of known plaintexts. In Section 4 we describe the single-key variant of the Even-Mansour scheme, which is strictly simpler but has the same level of provable security. In Section 5 we analyze the security of several other variants of the Even-Mansour scheme, demonstrating both the generality and the fragility of its formal proof of security. Another limitation of the proof technique is described in Section 6, where we show that no comparable lower bound on the memory complexity of our attacks can exist. Finally, in the Appendix we describe the mirror slide attack, which is a generalization of the slidex attack.

\footnotetext{
${ }^{1}$ We note that for specific block cipher structures, e.g., Feistel networks, a dedicated slide attack can require fewer than $2^{n / 2}$ plaintexts. However, there is no such method that works for general structures.
} 


\section{The Even-Mansour Scheme}

In this section we present the Even-Mansour (EM) scheme, review its security proof given in 9] and describe previous attacks on it presented in [5] and [4].

\subsection{Definition of the EM Scheme and Its Notation}

The Even-Mansour scheme is a block cipher which consists of a single publicly known permutation $\mathcal{F}$ over $n$-bit strings, preceded and followed by $n$-bit whitening keys $K_{1}$ and $K_{2}$, respectively, i.e.,

$$
E M_{K_{1}, K_{2}}^{\mathcal{F}}(P)=\mathcal{F}\left(P \oplus K_{1}\right) \oplus K_{2}
$$

It is assumed that the adversary is allowed to perform two types of queries:

- Queries to a full encryption/decryption oracle, called an E-oracle, that computes either $E(P)=E M_{K_{1}, K_{2}}^{\mathcal{F}}(P)$ or $D(C)=\left(E M_{K_{1}, K_{2}}^{\mathcal{F}}\right)^{-1}(C)$.

- Queries to an $\mathcal{F}$-oracle, that computes either $\mathcal{F}(x)$ or $\mathcal{F}^{-1}(y)$.

The designers of EM considered two types of attacks. In the first type, called existential forgery attack, the adversary tries to find a new pair $(P, C)$ such that $E(P)=C$. The second type is the more standard security game, where the adversary tries to decrypt a message $C$, i.e., to find $P$ for which $E(P)=C 2$ The data complexity of an attack on the scheme is determined by the number $D$ of queries to the $E$-oracle and their type (i.e., known/chosen/adaptively chosen etc.), and the time complexity of the attack is lower bounded by the number $T$ of queries to the $\mathcal{F}$-oracle 3 The success probability of an attack is the probability that the single guess it produces (either a pair $(P, C)$ for the first type of attack, or a plaintext $P$ for the second type) is correct.

\subsection{The Lower Bound Security Proof}

The main rigorously proven result in [9] was an upper bound of $O\left(D T / 2^{n}\right)$ on the success probability of any cryptanalytic attack (of either type) on EM that uses at most $D$ queries to the $E$-oracle and $T$ queries to the $\mathcal{F}$-oracle. This result implies that in order to attack EM with a constant probability of success, we must have $D T=\Omega\left(2^{n}\right)$. Since this security proof is crucial for some of our results, we briefly describe its main steps.

${ }^{2}$ These security notions are significantly different than the indistinguishability notions of [12] which proved similar lower bounds on the inability of the adversary to distinguish the given instance of the cipher from a random permutation. Finding the actual keys not only allows distinguishing the construction from a random permutation, but also allows winning the two security games considered in 9 .

${ }^{3}$ In concrete implementations, this oracle is usually replaced by some publicly known program which the attacker can run on its own. In this case the type of query (e.g., whether the inputs are adaptively chosen or not) can determine whether the attack can be parallelized on multiple processors, but we ignore such low level details in our analysis. 
The proof requires several definitions. Consider a cryptanalytic attack on EM, and assume that at some stage of the attack, the adversary already performed $s$ queries to the $E$-oracle and $t$ queries to the $\mathcal{F}$-oracle, and obtained sets $\mathcal{S}$ and $\mathcal{T}$ of $E$-pairs and $\mathcal{F}$-pairs, respectively, i.e.,

$$
\mathcal{D}=\left\{\left(P_{i}, C_{i}\right)\right\}_{i=1, \ldots, d}, \quad \text { and } \quad \mathcal{T}=\left\{\left(X_{j}, Y_{j}\right)\right\}_{j=1, \ldots, t} .
$$

We say that the key $K_{1}$ is bad with respect to the sets of queries $\mathcal{D}$ and $\mathcal{T}$, if there exist $i, j$ such that $P_{i} \oplus K_{1}=X_{j}$. Otherwise, $K_{1}$ is good with respect to $\mathcal{D}, \mathcal{T}$. Intuitively, a good key is one whose feasibility can not be deduced from the available data, whereas a bad key is one whose feasibility has to be analyzed further (but not necessarily discarded). Similarly, $K_{2}$ is bad w.r.t. $\mathcal{D}, \mathcal{T}$ if there exist $i, j$ such that $Y_{j} \oplus K_{2}=C_{i}$, and $K_{2}$ is good otherwise. The key $K=\left(K_{1}, K_{2}\right)$ is good with respect to $\mathcal{D}, \mathcal{T}$ if both $K_{1}$ and $K_{2}$ are good. It is easy to show that the number of good keys w.r.t. $\mathcal{D}$ and $\mathcal{T}$ is at least $2^{2 n}-2 s t \cdot 2^{n}$. A pair $\left(K=\left(K_{1}, K_{2}\right), \mathcal{F}\right)$ is consistent w.r.t. $\mathcal{D}$ and $\mathcal{T}$ if for any pair $\left(P_{i}, C_{i}\right) \in \mathcal{D}$ we have $C_{i}=K_{2} \oplus \mathcal{F}\left(P_{i} \oplus K_{1}\right)$, and for any pair $\left(X_{j}, Y_{j}\right) \in \mathcal{T}$, we have $\mathcal{F}\left(X_{j}\right)=Y_{j}$.

The proof consists of two main steps.

1. The first step shows that all good keys are, in some sense, equally likely to be the correct key. Formally, if the probability over the keys and over the permutations is uniform, then for all $\mathcal{D}, \mathcal{T}$, the probability

$$
\operatorname{Pr}_{K, \mathcal{F}}[K=k \mid(K, \mathcal{F}) \text { is consistent with } \mathcal{D}, \mathcal{T}]
$$

is the same for any key $k \in\{0,1\}^{2 n}$ that is good with respect to $\mathcal{D}, \mathcal{T}$.

We present the proof of this step, since it will be crucial in the sequel. It follows from Bayes' formula that it suffices to prove that the probability

$$
p=\operatorname{Pr}_{K, \mathcal{F}}[(K, \mathcal{F}) \text { is consistent with } \mathcal{D}, \mathcal{T} \mid K=k]
$$

is the same for all good keys. Given a good key $k=\left(k_{1}, k_{2}\right)$, it is possible to transform the set $\mathcal{D}$ of $E$-pairs to an equivalent set $\mathcal{D}^{\prime}$ of $\mathcal{F}$-pairs by transforming the $E$-pair $\left(P_{i}, C_{i}\right)$ to the $\mathcal{F}$-pair $\left(P_{i} \oplus k_{1}, C_{i} \oplus k_{2}\right)$. Since the key $k$ is good, the pairs in $\mathcal{D}^{\prime}$ and $\mathcal{T}$ do not overlap, and hence $p$ is simply the probability of consistency of a random permutation $\mathcal{F}$ with $d+t$ given distinct input/output pairs. This probability clearly does not depend on $k$, which proves the assertion.

2. The second step shows that the success probability of any attack is bounded by the sum of the probability that in some step of the attack, the right key becomes a bad key, and the probability that the adversary can successfully generate a "new" consistent $E$-pair $(P, C)$ if the right key is still amongst the good keys. The first probability can be bounded by $4 D T /\left(2^{n}-2 D T\right)$, and the second probability can be bounded by $1 /\left(2^{n}-D-T\right)$. Hence, the total success probability of the attack is bounded by $O\left(D T / 2^{n}\right)$. We omit the proof of this step since it is not used in the sequel. 
We note that obtaining non-trivial information about the key (e.g., that the least significant bit of the $K_{1}$ is zero, or the value of $K_{1} \oplus K_{2}$ ), is also covered by this proof. Hence, throughout the paper we treat such leakage of information as a "problem" in the security of the construction (even if the exact keys are not found).

Finally, we note that in 12 a slightly different model is considered. The analyzed construction is a one where besides the pre-/post-whitening keys, the internal permutation $\mathcal{F}$ is keyed with a $k$-bit key. For such a construction, Kilian and Rogaway prove that given $D$ queries to the construction and time $T$ evaluations of $\mathcal{F}$, one cannot succeed in distinguishing the construction from a random permutation with probability higher than $D T / 2^{n+k-1}$. Obviously, when $k=0$, i.e., the internal permutation is fixed, one can view this as a proof that indeed the Even-Mansour is indistinguishable from a random permutation with success rate over $D T / 2^{n-1}$. Note that in this paper we consider the stronger notion of attack (namely, finding the actual keys) and the thus the results are not identical.

\subsection{Previous Attacks on the Even-Mansour Scheme}

The first proposed attack on the Even-Mansour scheme was published by Joan Daemen at Asiacrypt 1991 [5]. Daemen used the framework of differential cryptanalysis 2 to develop a chosen plaintext attack which matched the Even-Mansour lower bound for any amount of given data. The approach is to pick $D$ pairs of chosen plaintexts whose XOR difference is some nonzero constant $\Delta$. This plaintext difference is preserved by the XOR with the prewhitening key $K_{1}$, and similarly, the ciphertext difference is preserved by the XOR with the postwhitening key $K_{2}$. For a known permutation $\mathcal{F}$, most combinations of input and output differences suggest only a small number of possible input and output values, but it is not easy to find them. To carry out the attack, all we have to do is to sample $2^{n} / D$ pairs of inputs to $\mathcal{F}$ whose difference is $\Delta$, and with constant non-negligible probability we can find an output difference which already exists among the chosen data pairs. This equality suggests actual input and output values to/from $\mathcal{F}$ for that pair, and thus recovers the two keys. We note that a similar chosen-plaintext attack was suggested in [12] for constructions where $\mathcal{F}$ is keyed (where $D T \geq 2^{n+k-1}$ for a $k$-bit keyed $\mathcal{F}$ ).

This attack matches the time/data relationship of the lower bound, but it is not tight since it requires chosen plaintexts, whereas the lower bound allows known plaintexts. This discrepancy was handled ten years later by a new attack called slide with a twist which was developed by Alex Biryukov and David Wagner, and presented at Eurocrypt 2000 [4. By taking two Even-Mansour encryptions, sliding one of them and reversing the other, they showed how to attack the scheme with known instead of chosen plaintexts 4 However, in order to find at least one slid pair, their attack requires at least $\Omega\left(2^{n / 2}\right)$ known plaintext/ciphertext pairs, and thus it could not be applied with a reasonable probability of success given any smaller number of known pairs.

${ }^{4}$ The slide with a twist attack on EM is described in detail in Section 3.1 
These two cryptanalytic attacks were thus complementary: One of them matched the full time/data tradeoff curve but required chosen plaintexts, while the other could use known plaintexts but only if at least $\Omega\left(2^{n / 2}\right)$ of them were given. In the next section we present the new slidex technique that closes this gap: it allows to use any number of known plaintexts with the same time/data tradeoff as in the lower bound proof, thus providing an optimal attack on the Even-Mansour scheme.

\section{The Slidex Attack and a Tight Bound on the Security of the Even-Mansour Scheme}

In this section we present the new Slidex attack and use it to obtain a tight bound on the security of the Even-Mansour scheme. We start with a description of the slide with a twist attack on EM 4 which serves as a basis for our attack, and then we present the slidex technique and apply it to EM. For more information on slide attacks, we refer the reader to 134 .

\subsection{The Slide with a Twist Attack}

The main idea of the slide with a twist attack on EM is as follows. Assume that two plaintexts $P, P^{*}$ satisfy

$$
P \oplus P^{*}=K_{1}
$$

In such a case, we have

$$
E(P)=\mathcal{F}\left(P \oplus K_{1}\right) \oplus K_{2}=\mathcal{F}\left(P^{*}\right) \oplus K_{2},
$$

and similarly,

$$
E\left(P^{*}\right)=\mathcal{F}\left(P^{*} \oplus K_{1}\right) \oplus K_{2}=\mathcal{F}(P) \oplus K_{2}
$$

(see Figure 1(a)). Hence,

$$
E(P) \oplus E\left(P^{*}\right)=\mathcal{F}(P) \oplus \mathcal{F}\left(P^{*}\right)
$$

or equivalently,

$$
E(P) \oplus \mathcal{F}(P)=E\left(P^{*}\right) \oplus \mathcal{F}\left(P^{*}\right) .
$$

This relation allows to mount the following attack:

1. Query both the $E$-oracle and the $\mathcal{F}$-oracle at the same $2^{(n+1) / 2}$ known values $P_{1}, P_{2}, \ldots 5$ Store in a hash table the pairs $\left.\left(E\left(P_{i}\right) \oplus \mathcal{F}\left(P_{i}\right)\right), i\right)$, sorted by the first coordinate.

2. For each collision in the table, i.e., $E\left(P_{i}\right) \oplus \mathcal{F}\left(P_{i}\right)=E\left(P_{j}\right) \oplus \mathcal{F}\left(P_{j}\right)$, check the guess $K_{1}=P_{i} \oplus P_{j}$ and $K_{2}=E\left(P_{i}\right) \oplus \mathcal{F}\left(P_{j}\right)$.

${ }^{5}$ Formally, the adversary obtains known plaintext/ciphertext pairs $\left(P_{i}, E\left(P_{i}\right)\right)$ and queries the $\mathcal{F}$-oracle at the value $P_{i}$. 
By the birthday paradox, it is expected that the data set contains a slid pair, i.e., a pair satisfying $P_{i} \oplus P_{j}=K_{1}$, with a non-negligible constant probability. For a random pair $\left(P_{i}, P_{j}\right)$, the probability that $E\left(P_{i}\right) \oplus \mathcal{F}\left(P_{i}\right)=E\left(P_{j}\right) \oplus \mathcal{F}\left(P_{j}\right)$ is $2^{-n}$, and thus, only a few collisions are expected in the table. These collisions include the collision induced by the slid pair, which suggests the correct values of $K_{1}$ and $K_{2}$. The data complexity of the attack is $D=2^{(n+1) / 2}$ known plaintexts, and the number of queries to $\mathcal{F}$ it requires is $T=2^{(n+1) / 2}$. Thus, $D T=2^{n+1}$, which matches the lower bound up to a constant factor of 2 .

\subsection{The New Slidex Attack}

The slidex attack is an enhancement of the slide with a twist technique, which makes it possible to use a smaller number of known plaintexts (i.e., queries to the $E$-oracle), in exchange for a higher number of queries to the $\mathcal{F}$-oracle. The basic idea of the attack is as follows: Assume that a pair of plaintexts $P, P^{*}$ satisfies

$$
P \oplus P^{*}=K_{1} \oplus \Delta
$$

for some $\Delta \in\{0,1\}^{n}$. In such a case,

$$
E(P)=\mathcal{F}\left(P \oplus K_{1}\right) \oplus K_{2}=\mathcal{F}\left(P^{*} \oplus \Delta\right) \oplus K_{2},
$$

and similarly,

$$
E\left(P^{*}\right)=\mathcal{F}\left(P^{*} \oplus K_{1}\right) \oplus K_{2}=\mathcal{F}(P \oplus \Delta) \oplus K_{2}
$$

(see Figure 1(b)). Hence,

$$
E(P) \oplus E\left(P^{*}\right)=\mathcal{F}\left(P^{*} \oplus \Delta\right) \oplus \mathcal{F}(P \oplus \Delta),
$$

or equivalently,

$$
E(P) \oplus \mathcal{F}(P \oplus \Delta)=E\left(P^{*}\right) \oplus \mathcal{F}\left(P^{*} \oplus \Delta\right)
$$

This allows to mount the following attack, for any $d \leq n$ :

1. Query the $E$-oracle at $2^{(d+1) / 2}$ arbitrary values (i.e., known plaintexts) $P_{1}, P_{2}, \ldots$..

2. Choose $2^{n-d}$ arbitrary values $\Delta_{1}, \Delta_{2}, \ldots$ of $\Delta$. For each $\Delta_{\ell}$, query the $\mathcal{F}$-oracle at the values $\left\{P_{i} \oplus \Delta_{\ell}\right\}_{i=1,2, \ldots, 2^{(d+1) / 2}}$, store in a hash table the pairs $\left.\left(E\left(P_{i}\right) \oplus \mathcal{F}\left(P_{i} \oplus \Delta_{\ell}\right)\right), i\right)$, sorted by the first coordinate, and search for a collision.

3. For each collision in any of the hash tables, i.e., when $P_{i}, P_{j}$ for which $E\left(P_{i}\right) \oplus \mathcal{F}\left(P_{i} \oplus \Delta_{\ell}\right)=E\left(P_{j}\right) \oplus \mathcal{F}\left(P_{j} \oplus \Delta_{\ell}\right)$ are detected, check the guess $K_{1}=P_{i} \oplus P_{j} \oplus \Delta_{\ell}$ and $K_{2}=E\left(P_{i}\right) \oplus \mathcal{F}\left(P_{j} \oplus \Delta_{\ell}\right)$.

For each triplet $\left(P_{i}, P_{j}, \Delta_{\ell}\right)$, the probability that $P_{i} \oplus P_{j} \oplus \Delta_{\ell}=K_{1}$ is $2^{-n}$. Since the data contains $2^{d} \cdot 2^{n-d}=2^{n}$ such triplets, it is expected that with a non-negligible constant probability the data contains at least one slidex triplet 
(a)

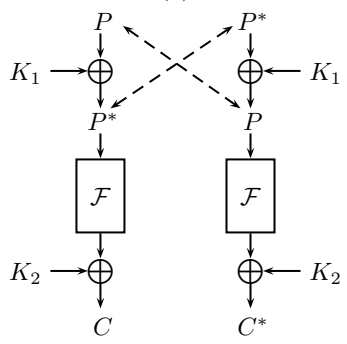

(b)

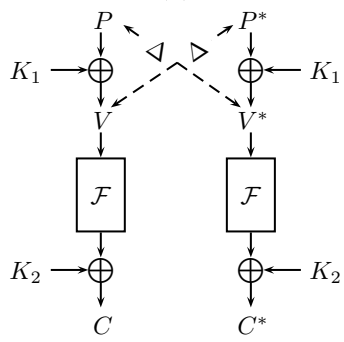

Fig. 1. (a) A twisted-slid pair; (b) A slidex pair

Table 1. Comparison of Results on the Even-Mansour scheme

\begin{tabular}{|c|c|c|}
\hline \multicolumn{3}{|c|}{ Known Plaintext Attacks } \\
\hline Attack & Data Time Memory & Tradeoff \\
\hline Guess and determine 9 & $2^{n}$ & - \\
\hline Slide with a twist 4 & $2^{n / 2} 2^{n / 2}$ & - \\
\hline Slidex (Sect. 3.2) & $D$ & $D T=2^{n}$ \\
\hline \multicolumn{3}{|c|}{ Chosen Plaintext Attacks } \\
\hline Attack & Data Time Memory & Tradeoff \\
\hline Differential [5] & $D$ & $D T=2^{n}$ \\
\hline \multicolumn{3}{|c|}{ Adaptive Chosen Plaintext Attacks } \\
\hline Attack & Data Time Memory & Tradeoff \\
\hline Slide (Sect. 6) & $D$ & $D T=2^{n}, D \geq 2^{n / 2}$ \\
\hline
\end{tabular}

(i.e., a triplet for which $\left.P_{i} \oplus P_{j} \oplus \Delta_{\ell}=K_{1}\right)$. On the other hand, since the probability of a collision in each hash table is $2^{d-n}$ and there are $2^{n-d}$ tables, it is expected that only a few collisions occur, and one of them suggests the correct key guess.

The number of queries to the $E$-oracle in the attack is $D=2^{(d+1) / 2}$, and the number of queries to the $\mathcal{F}$-oracle is $T=2^{n-(d-1) / 2}$. Thus, $D T=2^{n+1}$, which matches the lower bound of [9] up to a constant factor of 2 .

A summary of the complexities of all the old and new attacks on the Even-Mansour scheme appears in Table 1.

\section{The Single-Key Even-Mansour Scheme}

In this section we analyze the single-key variant of the Even-Mansour scheme (abbreviated in the sequel as "SEM"), which has the same level of security while using only $n$ secret key bits (compared to $2 n$ bits in EM) 6 First, we define the

${ }^{6}$ Kurosawa uses such SEMs in his constructions 13, where in each block the pre-/post-whitening keys are changed. 
scheme and show that the security proof of [9] can be adapted to yield a similar lower bound on its security. Then, we present a simple attack on the new scheme which matches the lower bound, thus proving its optimality.

\subsection{Definition of the Scheme and Its Security Proof}

Given a publicly known permutation $\mathcal{F}$ over $n$-bit strings and an $n$-bit secret key $K$, the Single-Key Even-Mansour (SEM) scheme is defined as follows:

$$
\operatorname{SEM}_{K}^{\mathcal{F}}(P)=\mathcal{F}(P \oplus K) \oplus K \text {. }
$$

The attack model is the same as in the EM scheme. That is, the adversary can query an encryption/decryption $E$-oracle and an $\mathcal{F}$-oracle, and the complexity of an attack is determined by the number $D$ of queries to the $E$-oracle and their type (known/chosen etc.), and the number $T$ of queries to the $\mathcal{F}$-oracle.

Surprisingly, the security proof of the EM scheme 9] holds almost without a change when we apply it to the single-key SEM variant. The only modification we have to make is to define a key $K$ as bad with respect to sets of oracle queries $S$ and $T$ if there exist $i, j$ such that either $P_{i} \oplus K=X_{j}$ or $C_{i} \oplus K=Y_{j}$, and $K$ as good otherwise. It is easy to see that if $|S|=s$ and $|T|=t$, then at least $2^{n}-2 s t$ keys are still "good" keys. Exactly the same proof as for EM shows that all the good keys are equally likely to be the right key, and the bounds on the success probability of an attack apply without change for SEM 7

Therefore, for any successful attack on SEM, we must have $D T=\Omega\left(2^{n}\right)$, which means that SEM provides the same security as EM, using only half as many key bits.

\subsection{A Simple Optimal Attack on SEM}

The slidex attack presented in Section 3 applies also to SEM, and is optimal since it uses only known plaintexts and matches everywhere the tradeoff curve of the security proof.

However, in the case of SEM, there is an even simpler attack (though, with the same complexity). Consider an encryption of a plaintext $P$ through SEM, and denote the intermediate values in the encryption process by:

$$
x=P, \quad y=P \oplus K, \quad z=\mathcal{F}(P \oplus K), \quad w=E(P)=\mathcal{F}(P \oplus K) \oplus K .
$$

Note that $x \oplus w=y \oplus z$. This allows to mount the following simple attack, applicable for any $D \leq 2^{n}$ :

1. Query the $E$-oracle at $D$ arbitrary values $P_{1}, P_{2}, \ldots, P_{D}$ and store in a hash table the values $\left(P_{i} \oplus E\left(P_{i}\right), i\right)$, sorted by the first coordinate.

\footnotetext{
${ }^{7}$ We note that the indistinguishability of this construction was also studied in 12, and it was shown that also the indistinguishability of SEM is the same as regular EM.
} 
2. Query the $\mathcal{F}$-oracle at $2^{n} / D$ arbitrary values $X_{1}, X_{2}, \ldots, X_{2^{n} / D}$, insert the values $X_{j} \oplus \mathcal{F}\left(X_{j}\right)$ to the hash table and search for a match.

3. If a match is found, i.e., $P_{i} \oplus E\left(P_{i}\right)=X_{j} \oplus \mathcal{F}\left(X_{j}\right)$, check the guess $K=P_{i} \oplus X_{j}$.

The analysis of the attack is exactly the same as that of the slide with a twist attack (see Section 3.1).

\section{The Security of Other Variants of the Even-Mansour Scheme}

In this section we consider two natural variants of the Even-Mansour scheme, and analyze their security.

The first variant replaces the XOR operations with modular additions, which are not involutions and are thus immune to standard slide-type attacks. However, we show that a new addition slidex attack can break it with the same complexity as that of the slidex attack on the original EM scheme.

The second variant considers the case in which the mapping $\mathcal{F}$ is chosen as an involution. This is motivated by the fact that in many "real-life" implementations of the EM scheme we would like to instantiate $\mathcal{F}$ by a keyless variant of a block cipher. Since in Feistel structures and many other schemes (e.g., KHAZAD, Anubis, Noekeon) the only difference between the encryption and decryption processes is the key schedule, such schemes become involutions when we make them keyless. In this section we show that this seemingly mild weakness of $\mathcal{F}$ can be used to mount a devastating attack on the EM scheme. In particular, we show that even when $\mathcal{F}$ is chosen uniformly at random among the set of all the possible involutions on $n$-bit strings, the adversary can recover the value $K_{1} \oplus K_{2}$ with $O\left(2^{n / 2}\right)$ queries to the $E$-oracle and no queries at all (!) to the $\mathcal{F}$-oracle. This clearly violates the lower bound proof that no significant information about the key can be obtained unless $D T=\Omega\left(2^{n}\right)$ (which was proven for random permutations but seems to be equally applicable to random involutions), and is achieved by a new variant of the slide attack, which we call the mirror slide attack.

\subsection{Even-Mansour with Addition}

Consider the following scheme:

$$
A E M_{K_{1}, K_{2}}^{\mathcal{F}}(P)=\mathcal{F}\left(P+K_{1}\right)+K_{2},
$$

where $\mathcal{F}$ is a publicly known permutation over $n$-bit strings, and ' + ' denotes modular addition in the additive group $Z_{2^{n}}$. In the sequel, we call it "Addition Even-Mansour" (AEM).

It is clear that the lower bound security proof of EM holds without any change for AEM. Similarly, it is easy to see that Daemen's differential attack on EM [5] can be easily adapted to AEM, by replacing XOR differences with modular differences. 
It may seem that the new variant has better security with respect to slide-type attacks. As noted in 4], ordinary slide attacks can be applied only for ciphers in which the secret key is inserted through a symmetric operation such as XOR, and not through modular addition. In the specific case of EM, the slide with a twist attack relies on the observation that if for two plaintexts $P, P^{*}$, we have $P^{*}=P \oplus K_{1}$, then surely, $P=P^{*} \oplus K_{1}$ as well. This observation fails for AEM: If $P^{*}=P+K_{1}$, then $P^{*}+K_{1}=P+2 K_{1} \neq P$ (unless $K_{1}=0$ or $K=2^{n-1}$ ). The slidex attack presented in Section 3.2 fails against AEM for the same reason. Hence, it seems that none of the previously known attacks can break AEM in the known plaintext model.

We present an extension of the slidex attack, which we call addition slidex, which can break AEM with data complexity of $D$ known plaintexts and time complexity of $T \mathcal{F}$-oracle queries, for any $D, T$ such that $D T=2^{n}$, hence showing that the security of AEM is identical to that of EM.

The basic idea of the attack is as follows: Assume that a pair of plaintexts $P, P^{*}$ satisfies $P+P^{*}=-K_{1}+\Delta$. (Note that somewhat counter intuitive, we consider the modular sum of the plaintexts rather than their modular difference!). In such a case,

$$
E(P)=\mathcal{F}\left(P+K_{1}\right)+K_{2}=\mathcal{F}\left(-P^{*}+\Delta\right)+K_{2},
$$

and similarly,

$$
E\left(P^{*}\right)=\mathcal{F}\left(P^{*}+K_{1}\right)+K_{2}=\mathcal{F}(-P+\Delta)+K_{2} .
$$

Hence,

$$
E(P)-E\left(P^{*}\right)=\mathcal{F}\left(-P^{*}+\Delta\right)-\mathcal{F}(-P+\Delta),
$$

or equivalently,

$$
E(P)+\mathcal{F}(-P+\Delta)=E\left(P^{*}\right)+\mathcal{F}\left(-P^{*}+\Delta\right) .
$$

Equation (2) allows us to mount an attack similar to the slidex attack, with the only change that instead of the values $\left.\left(E\left(P_{i}\right) \oplus \mathcal{F}\left(P_{i} \oplus \Delta\right)\right), i\right)$, the adversary stores in the hash table the values $\left.\left(E\left(P_{i}\right)+\mathcal{F}\left(-P_{i}+\Delta\right)\right), i\right)$.

We note that actually, the slidex attack can be considered as a special case of the addition slidex attack, since the addition slidex attack clearly applies to modular addition in any group, and the XOR operation corresponds to addition in the group $Z_{2}$.

\subsection{Even-Mansour with a Random Involution as the Permutation}

Let Involutional Even-Mansour (IEM) be the following scheme:

$$
I E M_{K_{1}, K_{2}}^{\mathcal{I}}(P)=\mathcal{I}\left(P \oplus K_{1}\right) \oplus K_{2},
$$


where $\mathcal{I}$ is chosen uniformly at random amongst the set of involutions on $n$-bit strings. We present a new technique, which we call mirror slide, that allows to recover the value $K_{1} \oplus K_{2}$ using $2^{n / 2}$ queries to the $E$-oracle, and with no queries to the $\mathcal{I}$-oracle.

The idea of the technique is as follows. Consider two input/output pairs $(P, C),\left(P^{*}, C^{*}\right)$ for IEM. Assume that we have

$$
P \oplus C^{*}=K_{1} \oplus K_{2} .
$$

In such case,

$$
P \oplus K_{1}=C^{*} \oplus K_{2},
$$

and hence, since $\mathcal{I}$ is an involution,

$$
\mathcal{I}\left(P \oplus K_{1}\right)=\mathcal{I}^{-1}\left(C^{*} \oplus K_{2}\right) .
$$

However, by the construction we have

$$
C=\mathcal{I}\left(P \oplus K_{1}\right) \oplus K_{2}, \quad \text { and } \quad P^{*}=\mathcal{I}^{-1}\left(C^{*} \oplus K_{2}\right) \oplus K_{1},
$$

and thus,

$$
C \oplus K_{2}=P^{*} \oplus K_{1}
$$

or equivalently,

$$
P^{*} \oplus C=K_{1} \oplus K_{2}=P \oplus C^{*},
$$

where the last equality follows from Equation (3). Therefore, assuming that $P \oplus C^{*}=K_{1} \oplus K_{2}$, we must have:

$$
P \oplus C=P^{*} \oplus C^{*} .
$$

This allows to mount a simple attack, similar to the slide with a twist attack. In the attack, the adversary queries the $E$-oracle at $2^{(n+1) / 2}$ arbitrary values $P_{1}, P_{2}, \ldots$, and stores in a hash table the pairs $\left(E\left(P_{i}\right) \oplus P_{i}, i\right)$, sorted by the first coordinate. It is expected that only a few collisions exist, and that with a non-negligible probability, one of them results from a pair $\left(P_{i}, P_{j}\right)$, for which $P_{i} \oplus E\left(P_{j}\right)=K_{1} \oplus K_{2}$.

Therefore, the attack supplies the adversary with only a few possible values of $K_{1} \oplus K_{2}$, after performing $2^{(n+1) / 2}$ queries to the $E$-oracle and no queries at all to the $\mathcal{I}$-oracle. As we show later, the adversary cannot obtain $K_{1}$ or $K_{2}$ themselves (without additional effort or data), but at the same time, the adversary does learn a nontrivial information about the key, which contradicts the security proof of the original EM scheme.

We note that this is an example for the gap between the indistinguishability security notion and the cost of finding a key. Obviously, when $K_{1}=K_{2}$ is known (or when $K_{1} \oplus K_{2}$ is known), one can easily distinguish the single-key involution Even-Mansour (ISEM) from a random permutation using two adaptive queries with extremely high probability. At the same time, the lower bounds of the Even-Mansour security proof assure us that it is impossible to decrypt a ciphertext $C$ encrypted by single-key involution Even-Mansour without first obtaining $D T=O\left(2^{n}\right)$ (similar result holds with respect to the existential forgery attack of producing another valid plaintext/ciphertext pair). 
Where the Security Proof Fails. One may wonder, which part of the formal security proof fails when $\mathcal{F}$ is an involution. It turns out that the only part that fails is the argument in the first step of the proof showing that all good keys are equally likely to be the right key. Recall that in order to show this, one has to show that the probability

$$
p=\operatorname{Pr}_{K, \mathcal{F}}[(K, \mathcal{F}) \text { is consistent with } \mathcal{D}, \mathcal{T} \mid K=k]
$$

is the same for all good keys. In the case of EM, $p$ is shown to be the probability of consistence of a random permutation $\mathcal{F}$ with $d+t$ given distinct input/output pairs, which indeed does not depend on $k$ (since such pairs are independent). In the case of IEM, the input/output pairs may be dependent, since it may occur that an encryption query to the $E$-oracle results in querying $\mathcal{I}$ at some value $x$, while a decryption query to the $E$-oracle results in querying $\mathcal{I}^{-1}$ at the same value $x$. Since $\mathcal{I}$ is an involution, these queries are not independent and thus, the probability $p$ depends on whether such dependency has occurred, and this event does depend on $k$. An examination of the mirror slide attack shows that this property is exactly the one exploited by the attack.

It is interesting to note that in the single-key case (i.e., for SEM where $\mathcal{F}$ is an involution, which we denote by ISEM), such event cannot occur, as in order to query $\mathcal{I}$ and $\mathcal{I}^{-1}$ at the same value, one must query $E$ and $E^{-1}$ at the same value. Since in the single-key case, the entire construction is an involution, such two queries result in the same answer for any value of the secret key, and hence, do not create dependence on the key. It can be shown, indeed, that the security proof does hold for ISEM and yields the same security bound, thus showing that in the case of involutions, the single-key variant is even stronger than the original two-key variant! Moreover, it can be noticed that in the case of EM, after the adversary recovers the value $K_{1} \oplus K_{2}$, the encryption scheme becomes equivalent to a single-key Even-Mansour scheme with the key $K_{1}$, i.e., $E^{\prime}(P)=\mathcal{I}\left(P \oplus K_{1}\right) \oplus K_{1}$. Thus, using two different keys in this case is totally obsolete, and also creates a security flaw which can be deployed by an adversary if the keys $K_{1}$ and $K_{2}$ are used also in other systems.

\subsection{Addition Even-Mansour with an Involution as the Permutation}

In this subsection we consider a combination of the two variants discussed in the previous subsections, i.e., AEM where $\mathcal{F}$ is a random involution. We abbreviate this variant as AIEM.

It can be easily shown that the mirror slide attack can be adapted to the case of AIEM, by modifying the assumption to $C^{*}-P=K_{1}+K_{2}$, and the conclusion to $P+C=P^{*}+C^{*}$. The attack allows to recover the value $K_{1}+K_{2}$, and then the scheme becomes equivalent to a conjugation EM scheme with a single key: $\operatorname{CISEM}(P)=\mathcal{I}\left(P+K_{1}\right)-K_{1}$, and it can be shown that the security proof of EM applies also to CISEM. Thus, the security of AEM under the assumption that $\mathcal{F}$ is an involution is identical to that of the original EM. 
An interesting phenomenon is that in the involution case, the security of single-key AEM (which we denote by AISEM) is much worse than that of AIEM. Indeed, the mirror slide attack allows to recover $K_{1}+K_{1}=2 K_{1}$, and hence to find $K_{1}$ (up to the value of the MSB), which breaks the scheme completely. This suggests that in the case of addition, the "natural" variant of single-key AEM is the conjugation variant, i.e., $\operatorname{CSEM}(P)=\mathcal{F}\left(P+K_{1}\right)-K_{1}$, for which the security proof of EM indeed applies even if $\mathcal{F}$ is an involution, as mentioned above.

In the extended version of this paper, available at [7, we consider all 12 variants of Even-Mansour (single key/two keys, random permutation/random involution, and whether the keys are XORed, added, or conjugated).

\section{Memoryless Attacks on the Even-Mansour Scheme}

All previous papers on the Even-Mansour scheme, including the lower bounds proved by the designers 9, Daemen's attack [5], and Biryukov-Wagner's slide attack 4], considered only the data and time complexities of attacks, but not the memory complexity. Analysis of the previously proposed attacks shows that in all of them, the memory complexity is $\min \{D, T\}$, where $D$ is the data complexity (i.e., the number of $E$-queries) and $T$ is the time complexity (i.e., the number of $\mathcal{F}$-queries). Thus, it is natural to ask whether the memory complexity can also be inserted into the lower bound security proofs, e.g., in the form $M \geq \min (D, T)$.

In this section we show that such a general lower bound can not exist, by constructing an attack with the particular data and time complexities of $O\left(2^{n / 2}\right)$, and with only a constant memory complexity. The attack is a memoryless variant of the slide with a twist attack described in Section 3.1. Recall that the main step of the slide with a twist attack is to find collisions of the form $E(P) \oplus \mathcal{F}(P)$ $=E\left(P^{*}\right) \oplus \mathcal{F}\left(P^{*}\right)$.

We observe that such collisions can be found in a memoryless manner. We treat the function

$$
\mathcal{G}: P \rightarrow E(P) \oplus \mathcal{F}(P)
$$

as a random function, and apply Floyd's cycle finding algorithm [10] (or any of its variants, such as Nivasch's algorithm [14) to find a collision in $\mathcal{G}$. The attack algorithm is as follows:

1. Query the $E$-oracle at a sequence of $O\left(2^{n / 2}\right)$ adaptively chosen values $P_{1}, P_{2}, \ldots$, such that $P_{1}$ is arbitrary and for $k>1, P_{k}=E\left(P_{k-1}\right) \oplus \mathcal{F}\left(P_{k-1}\right)$. (Here, after each query to the $E$-oracle, the adversary queries the $\mathcal{F}$-oracle at the same value and uses its answer in choosing the next query to the E-oracle).

2. Use Floyd's cycle finding algorithm to find $P_{i}, P_{j}$ such that $E\left(P_{i}\right) \oplus \mathcal{F}\left(P_{i}\right)$ $=E\left(P_{j}\right) \oplus \mathcal{F}\left(P_{j}\right)$.

3. For each colliding pair, check the guess $K_{1}=P_{i} \oplus P_{j}$ and $K_{2}=E\left(P_{i}\right) \oplus$ $\mathcal{F}\left(P_{j}\right)$. 
The analysis of the attack is identical to the analysis of the slide with a twist attack. The memory complexity is negligible, and the data and time complexities remain $O\left(2^{n / 2}\right)$. As the attack algorithm succeeds once a pair $P_{i}, P_{j}$ satisfying $E\left(P_{i}\right) \oplus \mathcal{F}\left(P_{i}\right)=E\left(P_{j}\right) \oplus \mathcal{F}\left(P_{j}\right)$ is found, the expected number of queries is determined by the random function $G$ 's graph. The analysis of graphs induced by random functions such as $G$ shows that the expected number of queries in the tail (the steps until entering the cycle) is $\pi m / 8$ and the length of the cycle itself is $\pi m / 8$ [14]. We note that these incur a small overhead in terms of query complexity (up to a factor of 5 in the case of Floyd's algorithm or 2 in the case Nivasch's cycle finding algorithm of [14] is used in exchange for a logarithmic memory). The only downside of this algorithm is the fact that the queries to the $E$-oracle are chosen adaptively, whereas in the slide with a twist attack we could choose arbitrary queries to the $E$-oracle.

\section{Open Problems}

If the amount of available $E$-oracle queries is smaller than $2^{n / 2}$, the adversary can still apply the slidex attack described in Section 3.2 but there seems to be no way to convert it into a memoryless attack by using the strategy described above. The main obstacle is that the adversary has to reuse the data many times in order to construct the hash tables for different values of $\Delta$, which can be done only if the data is stored somewhere rather than used in an on-line manner which discards it after computing the next plaintext. This leads to the following open problem:

Problem 1. Does there exist a memoryless attack on the Even-Mansour scheme with $D E$-oracle queries and $2^{n} / D \mathcal{F}$-oracle queries, where $D \ll 2^{n / 2}$ ?

A similar question can be asked with respect to the Single-Key Even-Mansour scheme, where in addition to the slidex attack, the simple attack presented in Section 4.2 can also break the scheme when $D \ll 2^{n / 2}$. The attack of Section 4.2 can also be transformed to a memoryless attack, by defining a random function:

$$
\mathcal{H}(X)= \begin{cases}X \oplus E(X), & L S B(X)=1 \\ X \oplus \mathcal{F}(X), & L S B(X)=0\end{cases}
$$

and using Floyd's cycle finding algorithm to find a collision of $\mathcal{H}$. In the case when $D$ and $T$ are both close to $2^{n / 2}$, with a constant probability such collision yields a pair $\left(X_{1}, X_{2}\right)$ such that $X_{1} \oplus E\left(X_{1}\right)=X_{2} \oplus \mathcal{F}\left(X_{2}\right)$, concluding the attack. The problem is that if $D \ll 2^{n / 2}$, then with overwhelming probability, a collision in $\mathcal{H}$ is of the form $X_{1} \oplus \mathcal{F}\left(X_{1}\right)=X_{2} \oplus \mathcal{F}\left(X_{2}\right)$, which is not useful to the adversary. Therefore, we state an additional open problem:

Problem 2. Does there exist a memoryless attack on the Single-Key Even-Mansour scheme with $D E$-oracle queries and $2^{n} / D \mathcal{F}$-oracle queries, where $D \ll 2^{n / 2}$ ? 
If such memoryless attack can be found only for Single-Key EM and not for the ordinary EM, this will show that at least in some respect, the use of an additional key in EM does make the scheme stronger.

\section{References}

1. Biham, E., Dunkelman, O., Keller, N.: Improved Slide Attacks. In: Biryukov, A. (ed.) FSE 2007. LNCS, vol. 4593, pp. 153-166. Springer, Heidelberg (2007)

2. Biham, E., Shamir, A.: Differential Cryptanalysis of the Data Encryption Standard. Springer (1993)

3. Biryukov, A., Wagner, D.: Slide Attacks. In: Knudsen, L.R. (ed.) FSE 1999. LNCS, vol. 1636, pp. 245-259. Springer, Heidelberg (1999)

4. Biryukov, A., Wagner, D.: Advanced Slide Attacks. In: Preneel, B. (ed.) EUROCRYPT 2000. LNCS, vol. 1807, pp. 589-606. Springer, Heidelberg (2000)

5. Daemen, J.: Limitations of the Even-Mansour Construction. In: [11], pp. 495-498

6. Dinur, I., Dunkelman, O., Shamir, A.: Improved Attacks on GOST. Technical report, to appear (2011)

7. Dunkelman, O., Keller, N., Shamir, A.: Minimalism in Cryptography: The EvenMansour Scheme Revisited. Cryptology ePrint Archive, Report 2011/541 (2011), http://eprint.iacr.org/

8. Even, S., Mansour, Y.: A Construction of a Cipher From a Single Pseudorandom Permutation. In: [11], pp. 210-224

9. Even, S., Mansour, Y.: A Construction of a Cipher from a Single Pseudorandom Permutation. J. Cryptology 10(3), 151-162 (1997)

10. Floyd, R.W.: Nondeterministic Algorithms. J. ACM 14(4), 636-644 (1967)

11. Imai, H., Rivest, R.L., Matsumoto, T. (eds.): ASIACRYPT 1991. LNCS, vol. 739. Springer, Heidelberg (1993)

12. Kilian, J., Rogaway, P.: How to Protect DES Against Exhaustive Key Search (an Analysis of DESX). J. Cryptology 14(1), 17-35 (2001)

13. Kurosawa, K.: Power of a Public Random Permutation and Its Application to Authenticated Encryption. IEEE Transactions on Information Theory 56(10), 5366-5374 (2010)

14. Nivasch, G.: Cycle Detection Using a Stack. Inf. Process. Lett. 90(3), 135-140 (2004)

15. Rivest, R.L.: DESX. Never published (1984)

16. Russian National Bureau of Standards: Federal Information Processing StandardCryptographic Protection - Cryptographic Algorithm. GOST 28147-89 (1989)

\section{A The Mirror Slide Attack}

In this section we present the general framework of the mirror slide attack, that was presented in Section 5.2 in the special case of the Even-Mansour scheme. We show that the mirror slide attack generalizes the slide with a twist attack 4]. We apply the new technique to a 20-round variant of the block cipher GOST [16], other variants of the attack are considered in the extended version of the paper [7. 


\section{A.1 The General Framework}

The mirror slide attack applies to block ciphers that can be decomposed as a cascade of three sub-ciphers: $E=E_{2} \circ E_{1} \circ E_{0}$, where the middle layer $E_{1}$ is an involution, i.e., $E_{1}=\left(E_{1}\right)^{-1} 8$

Let $E$ be such a cipher, and assume that for two plaintext/ciphertext pairs $(P, C),\left(P^{*}, C^{*}\right)$, we have

$$
E_{0}(P)=E_{2}^{-1}\left(C^{*}\right)
$$

In such case, since $E_{1}$ is an involution,

$$
E_{1}\left(E_{0}(P)\right)=E_{1}^{-1}\left(E_{2}^{-1}\left(C^{*}\right)\right) .
$$

By the construction, this implies:

$$
E_{2}^{-1}(C)=E_{1}\left(E_{0}(P)\right)=E_{1}^{-1}\left(E_{2}^{-1}\left(C^{*}\right)\right)=E_{0}\left(P^{*}\right) .
$$

If Equation (4) holds (and thus, Equation (5) also holds, the pair $\left(P, P^{*}\right)$ is called a mirror slid pair.

The way to exploit mirror slid pairs in a cryptanalytic attack is similar to standard slide-type attacks [3]4: The adversary asks for the encryption of $2^{(n+1) / 2}$ known plaintexts $P_{1}, P_{2}, \ldots$ (where $n$ is the block size of $E$ ) and denotes the corresponding ciphertexts by $C_{1}, C_{2}, \ldots$. For each pair $\left(P_{i}, P_{j}\right)$, the adversary assumes that it is a mirror slid pair and tries to solve the system of equations:

$$
\left\{\begin{array}{l}
C_{j}=E_{2}\left(E_{0}\left(P_{i}\right)\right) \\
C_{i}=E_{2}\left(E_{0}\left(P_{j}\right)\right)
\end{array}\right.
$$

(which is equivalent to Equations (4) and (5) ). If $E_{0}$ and $E_{2}$ are "simple enough", the adversary can solve the system efficiently and recover the key material used in $E_{0}$ and $E_{2}$.

If the amount of subkey material used in $E_{0}$ and $E_{2}$ is at most $n$ bits (in total), it is expected that at most a few of the systems of equations generated by the $2^{n}$ plaintext pairs are consistent (since the equation system is a $2 n$-bit condition). One of them is the system generated by the mirror slid pair, which is expected to exist in the data with a constant probability since the probability of a random pair to be a mirror slid pair is $2^{-n}$. Hence, the adversary obtains only a few suggestions for the key, which contain the right key with a constant probability. If the amount of key material used in $E_{0}$ and $E_{2}$ is bigger than $n$ bits, the adversary can still find the right key, by enlarging the data set by a small factor and using key ranking techniques (exploiting the fact that the right key is suggested by all mirror slid pairs, while the other pairs suggest "random" keys).

The data complexity of the attack is $O\left(2^{n / 2}\right)$ known plaintexts, and its time complexity is $O\left(2^{n}\right)$ (assuming that the system of equations can be solved within constant time).

\footnotetext{
${ }^{8}$ We note that the attack can be applied also if $E_{1}$ has some other symmetry properties, as shown in the extended version of the paper.
} 
We note that the attack can be applied even when $E_{0}$ and $E_{2}$ are not "simple" ciphers using a meet-in-the-middle attack. If both $E_{0}$ and $E_{2}$ use $\kappa \leq n$ key bits at most, one can try and find the solutions to the above set of equations in time $\min \left\{O\left(2^{n+\kappa}\right), O\left(2^{n / 2+2 \kappa}\right)\right\}$ 9

\section{A.2 The Slide with a Twist Attack and an Application to 20-Round GOST}

The first special case of the mirror slide framework we consider is where in the subdivision of $E$, we have $E_{2}=$ Identity. In such case, the system of equations presented above is simplified to:

$$
\left\{\begin{array}{l}
C_{j}=E_{0}\left(P_{i}\right), \\
C_{i}=E_{0}\left(P_{j}\right) .
\end{array}\right.
$$

It turns out that in this case, the attack is reduced exactly to the slide with a twist attack presented in [4! (Though, in [4] the attack is described in a different way).

A concrete example of this case is a reduced-round variant of the block cipher GOST [16, that consists of the last 20 of its 32 rounds. It is well-known that the last 16 rounds of GOST compose an involution, and hence, this variant can be represented as $E=E_{1} \circ E_{0}$, where $E_{0}$ is 4-round GOST, and $E_{1}$ (which is the last 16 rounds of GOST) is an involution 10 As shown in [6], a 4-round variant of GOST can be broken with two plaintext/ciphertext pairs and time complexity of $2^{12}$ encryptions. Therefore, the mirror slide attack can break this 20-round variant of GOST with data complexity of $2^{33}$ known plaintexts (since the block size of GOST is 64 bits), and time complexity of $2^{65} \cdot 2^{12}=2^{77}$ encryptions.

We note that a similar attack was described in [4] using the slide with a twist technique, but only on a 20-round version of a modified variant of GOST called GOST $\oplus$ in which the key addition is replaced by XOR.

${ }^{9}$ One can either take all plaintext/ciphertext pairs and partially encrypt the plaintext under all $2^{\kappa}$ keys for $E_{0}$ and partially decrypt the ciphertext under all $2^{\kappa}$ keys for $E_{2}$ to find the mirror pairs. Another option is to try for each pair of plaintexts $\left(P_{i}, P_{j}\right)$ to solve the system

$$
\left\{\begin{array}{c}
E_{2}^{-1}\left(C_{j}\right)=E_{0}\left(P_{i}\right), \\
E_{2}^{-1}\left(C_{i}\right)=E_{0}\left(P_{j}\right)
\end{array}\right.
$$

which can be easily done in a meet-in-the-middle approach in time $2^{\kappa}$ for each $\left(P_{i}, P_{j}\right)$.

10 We note that due to the Feistel structure of GOST, we do not have $E_{1} \circ E_{1}=I d$, but rather $E_{1} \circ$ swap $\circ E_{1}=I d$. This can be handled easily by inserting swap to the left hand side of Equation (6). The same correction can be performed in the other Feistel constructions discussed in the sequel. 